\title{
Transcription factor Oct4 promotes osteosarcoma by regulating IncRNA AK055347
}

\author{
HONGWU FAN ${ }^{1}$, GUANGYAO LIU ${ }^{1}$, CHANGFU ZHAO ${ }^{1},{\text { XUEFENG } \text { LI }^{2} \text { and XIAOYU YANG }}^{3}$ \\ Departments of ${ }^{1}$ Orthopedics and ${ }^{2}$ Anesthesiology, China-Japan Union Hospital of Jilin University; ${ }^{3}$ Department of \\ Orthopedics, Orthopedics Hospital, The Second Hospital of Jilin University, Changchun, Jilin 130001, P.R. China
}

Received June 2, 2015; Accepted October 24, 2016

DOI: $10.3892 / \mathrm{ol} .2016 .5400$

\begin{abstract}
Osteosarcoma is the most common primary bone tumor in children and adolescents, typically presenting with a poor prognosis. Octamer-binding transcription factor 4 (Oct4) protein, encoded by the POU class 5 homeobox 1 gene, is important in maintaining self-renewal of pluripotent stem cells, and is closely associated with cancer. However, its role in osteosarcoma remains to be elucidated. The present study observed Oct4 was markedly increased in osteosarcoma cell lines and in human osteosarcoma tissue samples. Following Oct4 downregulation by small interfering RNA (siRNA) in osteosarcoma F5M2 cells, the cells exhibited significant decreases in proliferation and invasion ability, and an increase in cell apoptosis. Notably, downregulation of Oct4 decreased the expression of AK055347, a newly identified long noncoding RNA (lncRNA) in human tissues. The downregulation of AK055347 by siRNA resulted in a significant suppressive effect on proliferative and invasive ability, and promotion of cell apoptosis in osteosarcoma cells. Thus, the current study suggests Oct4 exerts a promoting effect in osteosarcoma, and identifies a novel lncRNA in osteosarcoma progression.
\end{abstract}

\section{Introduction}

Octamer-binding transcription factor 4 (Oct4) protein is encoded by POU class 5 homeobox 1 (POU5F1), and belongs to a small group of pluripotency transcription factors (1). Oct4 is a key component and is critical in the maintenance of stem cell pluripotency and proliferation $(2,3)$. It is overexpressed in multiple types of human tumor and has been demonstrated to be associated with cancer progression. For example, the expression of Oct 4 was indicated to be significantly correlated with early hepatocellular carcinoma recurrence (4). Oct4 has been identified as overexpressed in human embryonic carcinoma (5),

Correspondence to: Dr Xiaoyu Yang, Department of Orthopedics, Orthopedics Hospital, The Second Hospital of Jilin University, 218 Ziqiang Street, Changchun, Jilin 130001, P.R. China E-mail: yangxiaoyu_ju@126.com

Key words: AK055347, lncRNA, Oct4, osteosarcoma, POU5F1 prostate cancer (6), and bladder cancer cells, and been associated with enhanced migration and invasion (7). Oct4 was also demonstrated to promote cell cycle progression in esophageal carcinoma (8), promote colony formation of glioma cells (9), and increase transmigration capacity in melanoma cells (10). These previous studies suggest that deregulation and dysfunction of Oct 4 contribute to malignant establishment of a cancer stem cell phenotype (5).

Osteosarcoma tends to have a poor prognosis, and is the leading primary bone tumor in children and adolescents. Tumor stem cells are hypothesized to promote tumor initiation, relapse and metastasis. Thus, the poor prognosis of osteosarcoma likely results in failure of treatment to target the osteosarcoma stem cells (11). In previous studies investigating the role of Oct4 in osteosarcoma, Oct4 was overexpressed in stem-like cells isolated from the human osteosarcoma MNNG/HOS cell line (12) and promoted tumor formation when OCT4 was exogenously expressed in the human osteosarcoma cell line (11). Overexpression of imprinted tumor-suppressing subtransferable candidate 3, an apoptosis-associated gene, efficiently downregulated the expression of Oct4 as well as homeobox protein NANOG (NANOG) and SRY (sex determining region Y)-box 2 (Sox2), which are two members of the pluripotency transcription factor family, in tumor initiating cells, decreased the clone formation rate, and downregulated tumorigenesis in the MThFOB1.19 osteoblast cell line (13). This indicates Oct4 may be involved in tumor promotion. However, the role of Oct4 in osteosarcoma requires further investigation.

Long noncoding RNAs (lncRNAs) are functional RNAs longer than 200 nucleotides. Increasing evidence suggests that lncRNAs are involved in the regulation of diverse cellular processes, including regulation of gene expression, imprinting, chromatin modification, transcription and posttranslational processing, particularly in carcinogenesis and metastasis $(14,15)$. A recent study demonstrated that Oct 4 and Sox 2 were increased in the RWPE-1 immortalized human prostate cell line, which had concomitant increase of lncRNA H19. Notably, targeted suppression of H19 with small interfering RNA (siRNA) decreased Oct4 and Sox 2 gene expression. Conversely, overexpression of $\mathrm{H} 19$ notably increased gene expression of these two transcription factors (16). Oct4 and Sox 2 also had a marked co-upregulation with IncRNA SOX2OT in the NTERA2 human embryonic carcinoma stem cell line (17). 
Thus, the present study aimed to investigate the effect of Oct4 on the proliferation and invasion of osteosarcoma cells via silencing Oct4 expression using siRNA. The current study also investigated the possible association between Oct 4 and a number of lncRNAs.

\section{Materials and methods}

Reagents. 3-(4, 5-dimethylthiazol-2-yl)-2, 5-diphenyltetrazolium bromide (MTT) was purchased from Sigma-Aldrich (Merck Millipore, Darmstadt, Germany). Fetal bovine serum (FBS), Lipofectamine 2000, non-essential amino acids, RPMI-1640 medium, TRIzol, Annexin V-fluorescein isothiocyanate (FITC) apoptosis detection kit, and trypsin-EDTA solution were purchased from Thermo Fisher Scientific, Inc. (Waltham, MA, USA). Antibodies against Oct4 (ab109183) or GAPDH (ab181602) were purchased from Abcam (Cambridge, UK). Horseradish peroxidase-coupled secondary immunoglobulin G (sc-2004) was purchased from Santa Cruz Biotechnology, Inc. (Dallas, TX USA). Enhanced chemiluminescence reagent was purchased from Pierce (Thermo Fisher Scientific, Inc.). SOSP-9607, MG63 and F5M2 osteosarcoma cell lines and the hFOB1.19 osteoblast cell line were purchased from the American Type Culture Collection (Manassas, VA, USA).

Patients and sample collection. A total of 10 patients who received osteosarcoma surgery in The Second Hospital of Jilin University (Changchun, China) between January 2012 and May 2014 were selected. These patients did not receive adjuvant treatment, including chemotherapy or radiotherapy, prior to the surgery. Biopsies were obtained from the osteosarcoma tissues and normal tissues adjacent to the tumor during surgery. The diagnosis was confirmed by pathological examination of these tissues. All the patients provided written informed consent and the present study was in strict accordance with National Institutes of Health guidelines (18) and was approved by the ethics committee of The Second Hospital of Jilin University.

Cell culture. SOSP-9607, MG63 and F5M2 osteosarcoma cell lines and the hFOB1.19 osteoblast cell line were grown in RPMI-1640 medium, supplemented with heat-inactivated FBS $(10 \% \mathrm{v} / \mathrm{v}), 100 \mathrm{U} / \mathrm{ml}$ penicillin, $100 \mu \mathrm{g} / \mathrm{ml}$ streptomycin and non-essential amino acids $(1 \% \mathrm{v} / \mathrm{v})$, and cultured at $37^{\circ} \mathrm{C}$ in an atmosphere of $5 \% \mathrm{CO}_{2}$ with a relative humidity of $95 \%$.

Transient transfection of Oct4 and AK055347 siRNAs. The transfection of Oct4 and AK055347 siRNA into F5M2 cells was performed using Lipofectamine 2000 according to the manufacturer's protocols. A total of $0.25 \times 10^{6}$ cells were seeded into 6 -well plates and $0.5 \mu \mathrm{g}$ siRNA was used. For transfection, Lipofectamine 2000 and siRNA were added directly to cells, and subsequently incubated at $37^{\circ} \mathrm{C}$. The sequences of the siRNAs and scramble controls were as follows: 5'-GGA GGAAGCTGACAACAATG-3' for Oct4-1; 5'-TTCAGCCAA ACGACCATC-3' for Oct4-2; 5'-GTATTCAGCCAAACG ACCAT-3' for Oct4-3; 5'-GGAGCAATGCACAGATGGAA-3' for Oct4 scramble; 5'-CACCTGAGTTGAATGAGGATC TACT-3' for AK055347-1; 5'-GAGTTGAATGAGGATCTA
CTGTTAA-3' for AK055347-2; 5'-CACGTACATGTGCAC ACACTGTCTA-3' for AK055347-3; and 5'-CACGAGGTT TAAGAGTAGTCCTACT-3' for AK055347-scramble.

Cell proliferation assay. Cell proliferation was analyzed using the MTT assay as previously described (19). Briefly, 1,000 cells from each group were plated in 96-well microplates in $150 \mu \mathrm{l}$ of medium. Following culture to different time points (1-5 days), $20 \mu \mathrm{l}$ of MTT substrate $(5 \mathrm{mg} / \mathrm{ml})$ was added to each well, and the plates were incubated for an additional $4 \mathrm{~h}$. The medium was then removed, and the cells were solubilized in $150 \mu \mathrm{l}$ of dimethyl sulfoxide. The culture plates were then agitated for $15 \mathrm{~min}$ and the optical absorbance values were read at a wavelength of $490 \mathrm{~nm}$.

Invasion assay. Cell invasion was evaluated using the Transwell invasion assay. Three days following transfection, F5M2 cells were trypsinized and resuspended in FBS-free RPMI-1640 medium. A total of $2 \times 10^{4}$ cells were plated in the upper chamber of the Transwell with a Matrigel-coated polycarbonate membrane. Fresh medium with $10 \%$ FBS was added to the lower chamber as a chemoattractant. Following incubation for a further $48 \mathrm{~h}$ at $37^{\circ} \mathrm{C}$ with $5 \% \mathrm{CO}_{2}$, cells on the lower surface of the membrane were fixed with $5 \%$ formalin and stained with $0.2 \%$ crystal violet. The non-migrated cells on the upper surface were wiped off with a cotton swab. Images of the cells that migrated to the lower surface were captured. The invaded cells were counted in five randomly selected fields using a light microscope.

Flow cytometry analysis. Cell apoptosis was analyzed with an Annexin V-FITC apoptosis detection kit according to the manufacturer's protocols. Briefly, the cells were washed with cold phosphate-buffered saline (PBS) and collected with a trypsin-EDTA solution. The cell suspensions were then centrifuged at $95 \mathrm{x} \mathrm{g}$ for $5 \mathrm{~min}$ at $4^{\circ} \mathrm{C}$ to remove the trypsin-EDTA solution. The cells $\left(3 \times 10^{6} / \mathrm{ml}\right)$ were then re-suspended and incubated with Annexin V-FITC and Annexin V binding buffer for $15 \mathrm{~min}$ at room temperature. The stained cells were analyzed with a BD LSR II flow cytometer (BD Biosciences, San Jose, CA, USA).

Reverse transcription-quantitative polymerase chain reaction $(R T$ - $q P C R)$. RNA levels were measured using RT-qPCR as previously described (20). Tumor and adjacent normal tissue samples were used. Total RNA was extracted using TRIzol reagent according to the manufacturer's protocols. The RNA was reverse transcribed at $42^{\circ} \mathrm{C}$ for $1 \mathrm{~h}$ into cDNA using M-MLV reverse transcriptase (Thermo Fisher Scientific, Inc.) and random primers (Thermo Fisher Scientific, Inc.). PCR was performed using SYBR GreenER qPCR SuperMix Universal kit (Thermo Fisher Scientific, Inc.) with an ABI StepOnePlus Real-Time PCR system (Applied Biosystems; Thermo Fisher Scientific, Inc.). The thermocycling conditions used were as follows: Initial denaturation at $95^{\circ} \mathrm{C}$ for $5 \mathrm{~min}$; and 39 cycles of denaturation at $95^{\circ} \mathrm{C}$ for $30 \mathrm{sec}$, annealing at $60^{\circ} \mathrm{C}$ for $30 \mathrm{sec}$ and extension at $72^{\circ} \mathrm{C}$ for $45 \mathrm{sec}$. Specific primer sequences are listed in Table I. Results are presented as the levels of expression relative to those of the controls with GAPDH for normalization using the $2^{-\Delta \Delta \mathrm{Cq}}$ method (21). 
Table I. Primer sequences.

Gene

AK023948

AK027193

AK055347

BCAR4

CASC2

GAPDH

HIF1A-AS1

PCA3

POU5F1

PVT1

SNHG5
Forward (5'-3')

Reverse (5'-3')
TTTAGTTCCAATCTGTCCC GCTCTTGGGTGAAGAAAC AGGACCCACTGACTGAAC ACCTATGGCTATCATTGTT TCTGTGTTGATGTTGATT AGCCACATCGCTCAGACAC GTATGTCTCAGTTATCTTCCT TTAACAACTGGTCCTGAG CACTCGGTTCTCGATACTGG CAGATGAACCAGGTGAAC ACTAGCCAGAAATCGTTG

BCAR4, breast cancer anti-estrogen resistance 4; CASC2, cancer susceptibility candidate 2; HIF1A-AS1, HIF1A antisense RNA 1; PCA3, prostate cancer associated 3; POU5F1, POU class 5 homeobox 1; PVT1, Pvt1 oncogene; SNHG5, small nucleolar RNA host gene 5.
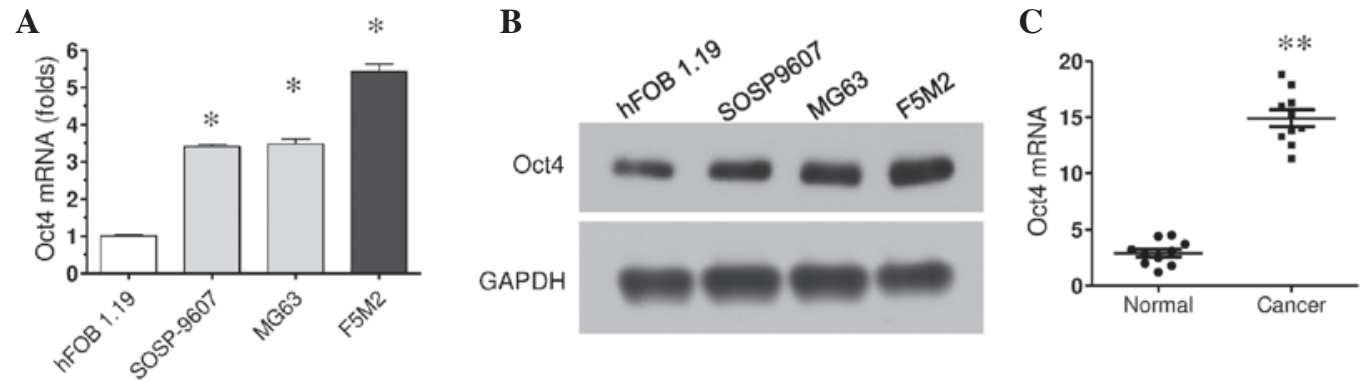

Figure 1. Oct4 expression in osteosarcoma tissues and cell lines. (A) mRNA and (B) protein expression in three osteosarcoma cell lines. (C) mRNA expression of Oct4 in osteosarcoma tissues and adjacent normal tissues $(n=10)$. mRNA and protein expression were measured using reverse transcription-quantitative polymerase chain reaction and western blotting. ${ }^{*} \mathrm{P}<0.05$ vs. hFOB1.19, ${ }^{* *} \mathrm{P}<0.01$ vs. the normal tissue. Oct 4 , octamer-binding transcription factor 4.

A

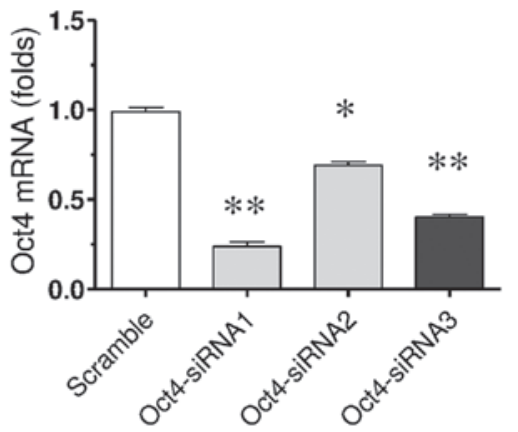

B

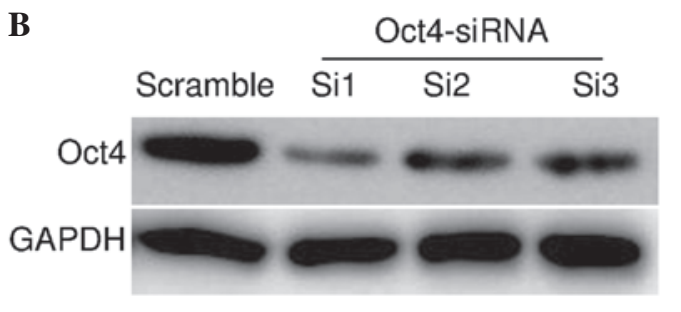

Figure 2. Oct4 siRNA reduces Oct4 expression in F5M2 cells. F5M2 cells were treated with Oct4 siRNA or scramble for 3 days. (A) mRNA and (B) protein expression levels were measured by reverse transcription-quantitative polymerase chain reaction and western blotting, respectively $(\mathrm{n}=6)$. ${ }^{*} \mathrm{P}<0.05$, ${ }^{* *} \mathrm{P}<0.01$ vs. the scramble group. Oct4, octamer-binding transcription factor 4; siRNA, small interfering RNA.

Western blot analysis. The protein expression levels of Oct4 in cells were assessed using western blotting as described in a previous study (20). Proteins were quantified by the BCA assay. Whole cell proteins $(2 \mu \mathrm{g})$ were separated electrophoretically in 4-12\% SDS-PAGE gels, and transferred to nitrocellulose membranes. Following 30 min of blocking with $2.5 \%$ nonfat milk, the membranes were incubated with primary antibodies $(1: 2,000)$ at $4^{\circ} \mathrm{C}$ overnight. Subsequently the membranes were incubated for $1 \mathrm{~h}$ at room temperature with horseradish peroxidase-conjugated secondary antibody $(1: 2,000)$. The membranes were then adequately washed with PBS containing $0.5 \%$ Tween 20 . The membranes were developed with enhanced chemiluminescence reagent (Pierce, Rockford, IL, USA) and then exposed to X-ray film.

Statistical analysis. Data are presented as the mean \pm standard error of the mean. Statistical analysis was performed using one-way analysis of variance followed by Bonferroni's 
A

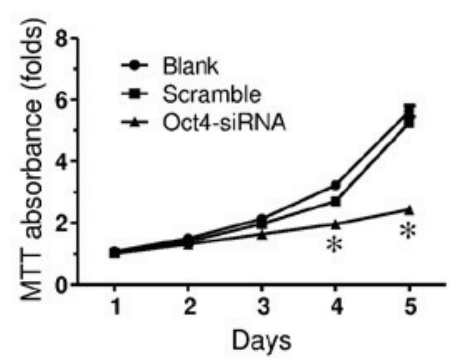

C

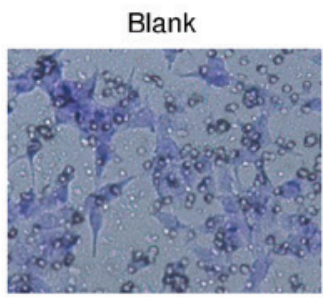

B

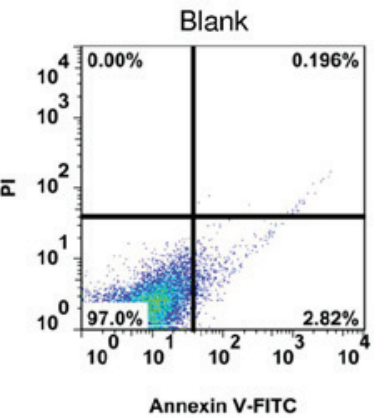

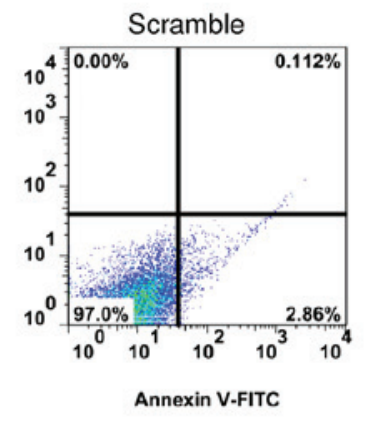

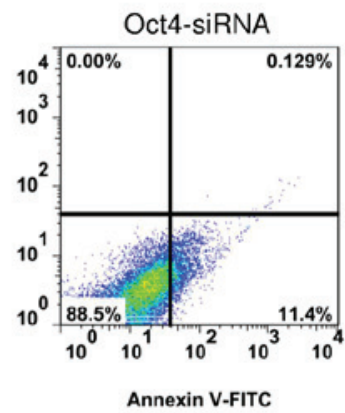

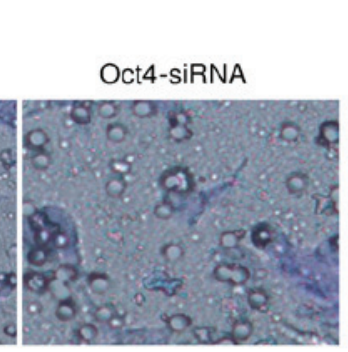

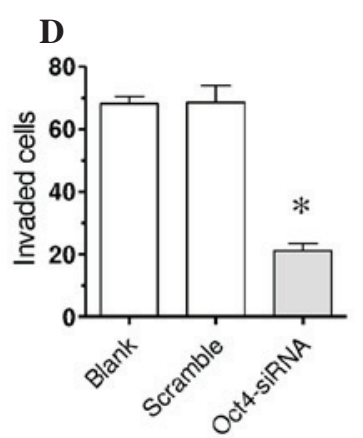

Figure 3. Oct4 downregulation decreases proliferation and invasion ability of F5M2 cells. F5M2 cells were treated with of Oct4 siRNA1 or scramble for 3 days. (A) Proliferation was measured by MTT assay, (B) cell apoptosis was assayed using Annexin V by flow cytometry, and (C) invasion ability was evaluated by Transwell assay. Magnification, x200. (D) Quantified data of invasion has been presented ( $\mathrm{n}=6$ ). * $\mathrm{P}<0.01$ vs. the control. Oct4, octamer-binding transcription factor 4; siRNA, small interfering RNA; FITC, fluorescien isothiocyanate; PI, propidium iodide.

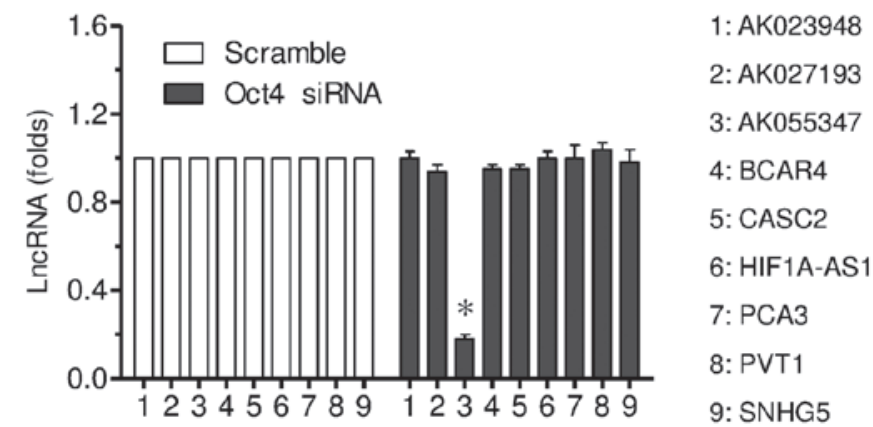

Figure 4. Effects of Oct4 downregulation on the expression levels of numerous lncRNAs. F5M2 cells were treated with Oct 4 siRNA1 or scramble for 3 days. The expressions of lncRNAs were measured by real-time RT-PCR $(n=6)$. ${ }^{*} \mathrm{P}<0.01$ vs. scramble. lncRNA, long non-coding RNA; siRNA, small interfering RNA; Oct4, octamer-binding transcription factor 4; BCAR4, breast cancer anti-estrogen resistance 4; CASC2, cancer susceptibility candidate 2 HIF1A-AS1, HIF1A antisense RNA 1; PCA3, prostate cancer associated 3; PVT1, Pvt1 oncogene; SNHG5, small nucleolar RNA host gene 5.

post-hoc test for multiple comparisons. $\mathrm{P}<0.05$ was considered to indicate a statistically significant difference. Statistical analysis was performed using SPSS version 19 (IBM SPSS, Armonk, NY, USA)

\section{Results}

Oct4 expression is increased in various osteosarcoma cell lines and human osteosarcoma tissues. Oct4 mRNA expression was investigated in various osteosarcoma cells using RT-qPCR. The results demonstrated that Oct4 expression was significantly increased in the three types of osteosarcoma cells, SOSP9607, MG63 and F5M2, compared with the hFOB1.19 osteoblast cell line ( $\mathrm{P}<0.05$; Fig. 1A). Of these cells, F5M2 cells exhibited the highest Oct4 expression levels. Oct4 protein expression levels in these cells were subsequently investigated using western blotting. Consistent with mRNA expression levels, the protein expression of Oct4 was also increased in these osteosarcoma cells compared with normal cells (Fig. 1B).

It was also further examined whether the increased Oct4 expression levels were also observed in human osteosarcoma tissues using RT-qPCR. The mRNA was extracted from cancer tissue samples and adjacent normal tissue samples from 10 patients with osteosarcoma, and RT-qPCR was performed to measure Oct4 mRNA expression levels. When compared with the normal tissues, Oct4 expression in cancer tissues was increased in each pair of samples, and significantly higher collectively $(\mathrm{P}<0.01$; Fig. $1 \mathrm{C})$. These results demonstrate POU5F1/Oct4 expression is increased in osteosarcoma, and suggest POU5F1/Oct4 is associated with osteosarcoma progression.

Downregulation of Oct4 suppresses cell proliferation and invasion. To investigate the possible role of increased Oct4 expression in osteosarcoma, F5M2 osteosarcoma cells were transfected with siRNA against Oct4 or scrambled RNA to downregulate Oct4 expression. After 3 days transient transfection with Oct4 siRNA, the mRNA and protein expression levels of Oct 4 was detected by RT-qPCR and western blotting (Fig. 2). The results demonstrated that the mRNA expression levels were significantly decreased by three different Oct4 siRNAs $(\mathrm{P}<0.05$ and $\mathrm{P}<0.01$; Fig. $2 \mathrm{~A})$. Among them, Oct4 


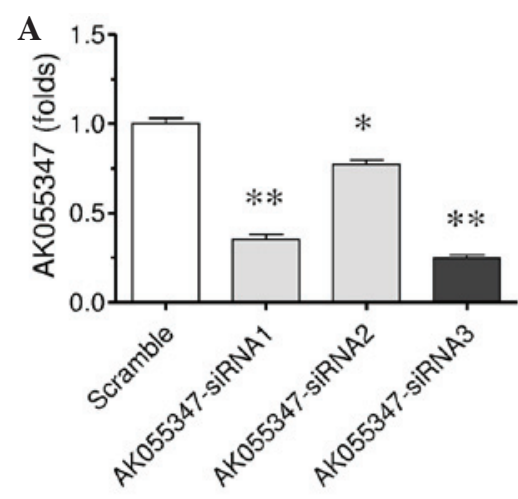

C

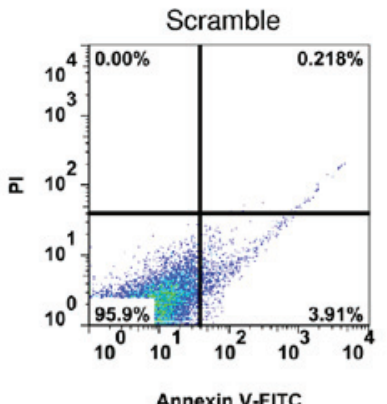

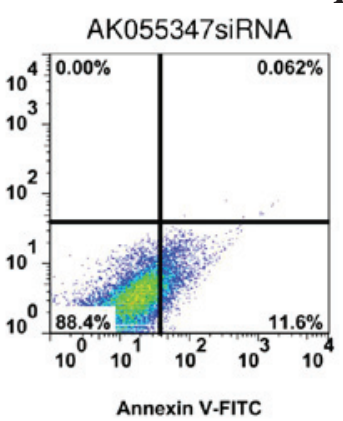

B

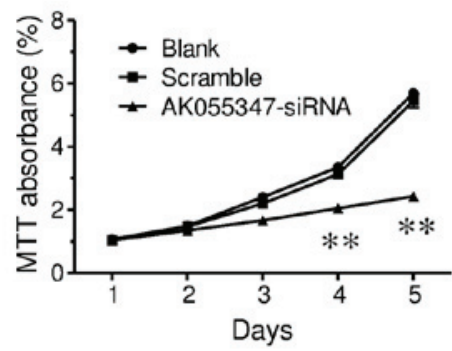

D

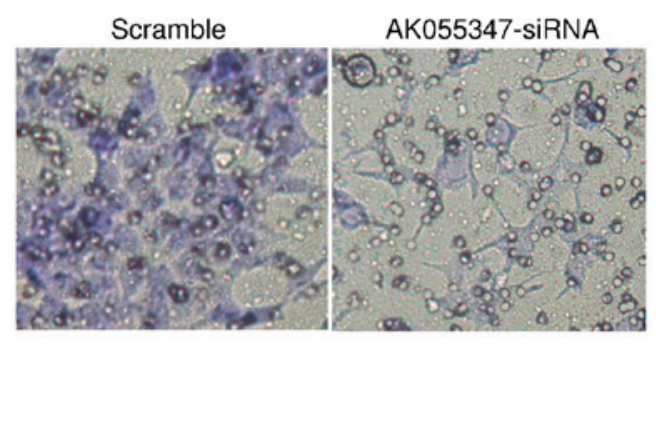

Figure 5. AK055347 downregulation decreases proliferation and invasion ability of F5M2 cells. F5M2 cells were treated with of AK055347 siRNA3 or scramble for 3 days. (A) Proliferation was measured by MTT assay, (B) cell apoptosis was assayed with Annexin V by flow cytometry and (C) invasion ability was evaluated by Transwell assay $(n=6) .{ }^{*} \mathrm{P}<0.05,{ }^{* *} \mathrm{P}<0.01$ vs. the scramble group. (D) AK055347 downregulation decreased cell invasion ability. Magnification, x200. siRNA, small interfering RNA; PI, propidium iodide; FITC, fluorescein isothiocyanate.

siRNA-1 revealed biggest inhibitive effect $(\mathrm{P}<0.01)$. This was consistent with the protein expression level results. Thus, Oct4 siRNA-1 was used in further experiments.

It was subsequently investigated whether the downregulation of Oct 4 affected the proliferation of F5M2 cells using the MTT assay. F5M2 cells were transfected with Oct4 siRNA-1 or scramble siRNA. In the MTT assay, Oct4 siRNA transfection significantly decreased cell proliferation in a time-dependent manner compared with the scramble control, which was indicated by decreased MTT absorbance value (Fig. 3A). The scramble control indicated a similar change in the MTT absorbance value when compared with the blank control. Furthermore, the current study investigated whether the decreased proliferation by downregulation of Oct4 was associated with cell apoptosis. Cell surface expression of Annexin $\mathrm{V}$ was measured using flow cytometry. As presented in Fig. 3B, scramble RNA did not markedly affect surface expression of Annexin V. Oct4 siRNA markedly increased the surface expression of Annexin V, suggesting increased apoptosis by Oct4 downregulation.

Invasion is an important characteristic of tumor metastasis. The present study examined whether Oct4 is involved in osteosarcoma metastasis using the Transwell invasion assay. In F5M2 cells with Oct4 downregulation, fewer cells invaded through the Matrigel-coated polycarbonate membrane as compared with the scramble control and blank control (Fig. 3C and D; $\mathrm{P}<0.01$ ). This indicates that Oct4 is involved in promotion of invasion of osteosarcoma cells.

Downregulation of Oct4 suppresses AK055347 expression levels. RT-qPCR was conducted to investigate the effect of
Oct4 downregulation on lncRNA expression. The current study observed downregulation of Oct 4 significantly decreased AK055347 expression, but not the other eight lncRNAs investigated $(\mathrm{P}<0.01$; Fig. 4).

Downregulation of AK055347 suppresses cells proliferation and invasion. To investigate whether the decreased expression of Ak055347 by Oct4 is the underlying mechanism of Oct4 in osteosarcoma, the effect of downregulated AK055347 on proliferation and invasion ability of F5M2 cells was investigated. F5M2 cells were treated with AK055347 siRNA or scramble control for 3 days. RT-qPCR was performed to validate the downregulation of AK055347 by three different AK055347 siRNAs $(\mathrm{P}<0.05$ and $\mathrm{P}<0.01$; Fig. 5A). Among them, AK055347 siRNA3 exhibited the most marked inhibitive effect $(\mathrm{P}<0.01)$. Thus, AK055347 siRNA-3 was used in further experiments.

Similarly to experiments for Oct4, the effect of AK055347 downregulation on cell proliferation, apoptosis and invasion was investigated. F5M2 cells were transfected with AK055347 siRNA-3 or scramble control, and the cells were cultured for 1-5 days, and the MTT assay was subsequently performed. The results indicated that AK055347 downregulation significantly decreased MTT absorbance value in a time-dependent manner as compared with the scramble control (Fig. 5B). AK055347 downregulation markedly increased the surface expression of Annexin V compared with the scramble control (Fig. 5C). Further, AK055347 downregulation decreased cell invasion ability in Transwell invasion assay (Fig. 5D). Thus, these results suggest AK055347 promotes cell proliferation and invasion, and has a suppressive effect on apoptosis. 


\section{Discussion}

The present study aimed to investigate the role of POU5F1/Oct4 in the regulation of osteosarcoma progression and elucidate possible underlying mechanisms. It was observed that Oct4 expression was significantly increased in various osteosarcoma cell lines and osteosarcoma tissues. Oct4 downregulation significantly decreased osteosarcoma cell proliferation and invasion ability, and increased cell apoptosis. The downregulation of Oct4 reduced the expression of AK055347, a novel identified lncRNA. AK055347, in a siRNA-mediated downregulation model, suggesting a role in tumor promotion in osteosarcoma cells. Thus, the results of the present study suggest Oct4 is involved in the promotion of osteosarcoma, which may be mediated via AK055347.

POU5F1/Oct4 is a recently identified proto-oncogene that serves as a marker of aggressive phenotypes in a number of types of cancer. In a previous study, strong expression of Oct4 was observed in recurrent prostate cancer when compared with adjacent normal tissue samples (6). In patients with head and neck squamous cell carcinoma, increased expression levels of Oct 4 , in addition to cytoplasmic/nuclear $\beta$-catenin, was considered to be associated with a worse prognosis (22). In the present study, the results indicated that Oct4 expression was increased in the osteosarcoma tissues obtained from 10 osteosarcoma patients compared with adjacent normal tissues. This finding is consistent with these previous studies $(6,22)$. Furthermore, in three types of osteosarcoma cells, SOSP-9607, MG63 and F5M2, the mRNA expression levels of Oct4 were significantly increased and protein expression levels were also increased compared with the hFOB1.19 osteoblast cell line. Thus, the results of the present study demonstrate that Oct4 is highly expressed in osteosarcoma.

In order to elucidate whether the increased Oct4 expression is associated with poor prognosis of osteosarcoma, Oct4 was downregulated by specific siRNA in the present study. The results indicated Oct 4 downregulation markedly reduced cell proliferation and induced cell apoptosis. In addition, the present study also demonstrated that Oct4 downregulation reduced cell invasion ability in a Transwell invasion assay. These results suggest a promotive role of Oct4 in osteosarcoma progress. Proliferation and invasion, in addition to migration, are important characteristics of cancer. Particularly, invasion is involved in cancer cell metastasis, which ultimately leads to a poor prognosis. The promotive effect of Oct4 on cancer cell proliferation was reported in certain types of cancer cells, including hepatocellular carcinoma (23) and esophageal cancer (8). The identification of Oct4-positive putative stem cells may result in uncontrolled cell proliferation and treatment failure in esophageal squamous cell carcinoma (24). The promoting effect of Oct4 on migration and invasion has also been observed in numerous types of cancer. In gastric cancer, only positive expression of Oct4, but not other proteins, including NANOG, Sox2, proliferating cell nuclear antigen, Ki67 and epithelial cadherin was reported to be correlated with lymphatic invasion (25). Oct4 was also reported to enhance lung cancer cell invasion and adhesion accompanied by the downregulation of epithelial marker cytokeratin and upregulation of the mesenchymal markers vimentin and neural cadherin (26). The percentage of OCT4-positive cells was significantly increased in higher grade, higher non-classic differentiation number and more invasive urothelial bladder cancer cases (27). Oct4 was also suggested to promote proliferation and invasion of esophageal cancer cells (8). The data in the present study further supports this role for Oct4 in cancer progression. Notably, a controversial effect of Oct4 was observed in breast cancer. Oct4 was determined to exert a suppressive effect on cell migration and invasion in vitro and the formation of metastatic lung nodules in vivo (28).

Alterations in the expression of a number of lncRNAs have been observed in certain types of cancer, and this has been indicated to be important in carcinogenesis and metastasis (14). Li et al (29) demonstrated that 25,733 lncRNAs were expressed in osteosarcoma tissue samples from a microarray analysis. Among them, 403 lncRNAs were consistently upregulated, while 798 lncRNAs were consistently downregulated in all samples analyzed. In the present study, AK055347, an IncRNA, was identified to be markedly decreased by Oct 4 downregulation in osteosarcoma cells. Previous studies have demonstrated that IncRNAs may exert different effects in osteosarcoma. Metastasis-associated lung adenocarcinoma transcript 1 was reported to promote the proliferation and metastasis of osteosarcoma cells (30). Downregulation of taurine upregulated 1 inhibited osteosarcoma cell proliferation and promoted apoptosis (31). Genetic deletions of LOC285194 and $\mathrm{BC} 040587$ were determined to be associated with poor survival of osteosarcoma patients (32). These lncRNAs were indicated to promote osteosarcoma progress. By contrast, a novel lncRNA, hypoxia-inducible factor- $2 \alpha$ promoter upstream transcript, was indicated to function as an inhibitor of osteosarcoma stem cells in vitro (33). In the present study, AK055347 downregulation markedly decreased cell proliferation, increased apoptosis and reduced invasion ability. Thus, the data suggests that AK055347, similar to the majority of identified lncRNAs, has a promotive effect on osteosarcoma progression.

In conclusion, the results presented in the present study demonstrate increased expression of POU5F1/Oct4 in osteosarcoma, which may exert a promotive effect in osteosarcoma progression via regulating AK055347 expression levels. These findings provide potential targets for clinical applications.

\section{References}

1. Boyer LA, Lee TI, Cole MF, Johnstone SE, Levine SS, Zucker JP, Guenther MG, Kumar RM, Murray HL, Jenner RG, et al: Core transcriptional regulatory circuitry in human embryonic stem cells. Cell 122: 947-956, 2005.

2. Bi Y, He Y, Huang J, Su Y, Zhu GH, Wang Y, Qiao M, Zhang BQ, Zhang H, Wang Z, et al: Functional characteristics of reversibly immortalized hepatic progenitor cells derived from mouse embryonic liver. Cell Physiol Biochem 34: 1318-1338, 2014.

3. Zeineddine D, Hammoud AA, Mortada M and Boeuf H: The Oct4 protein: More than a magic stemness marker. Am J Stem Cells 3: 74-82, 2014.

4. Chang TS, Wu YC, Chi CC, Su WC, Chang PJ, Lee KF, Tung TH, Wang J, Liu JJ, Tung SY, et al: Activation of IL6/IGFIR confers poor prognosis of HBV-related hepatocellular carcinoma through induction of Oct4/NANOG expression. Clin Cancer Res 21: 201-210, 2015.

5. Park SW, Do HJ, Ha WT, Han MH, Park KH, Song H, Kim NH and Kim JH: Transcriptional activation of Oct4 by the ETS transcription factor PEA3 in NCCIT human embryonic carcinoma cells. FEBS Lett 588: 3129-3136, 2014. 
6. Guzel E, Karatas OF, Duz MB, Solak M, Ittmann M and Ozen M Differential expression of stem cell markers and ABCG2 in recurrent prostate cancer. Prostate 74: 1498-1505, 2014.

7. Chang CC, Shieh GS, Wu P, Lin CC, Shiau AL and Wu CL: Oct-3/4 expression reflects tumor progression and regulates motility of bladder cancer cells. Cancer Res 68: 6281-6291, 2008

8. Li Z, Li X, Li C, Su Y, Fang W, Zhong C, Ji W, Zhang Q and $\mathrm{Su}$ C: Transcription factor OCT4 promotes cell cycle progression by regulating CCND1 expression in esophageal carcinoma. Cancer Lett 354: 77-86, 2014.

9. Du Z, Jia D, Liu S, Wang F, Li G, Zhang Y, Cao X, Ling EA and Hao A: October4 is expressed in human gliomas and promotes colony formation in glioma cells. Glia 57: 724-733, 2009.

10. Borrull A, Ghislin S, Deshayes F, Lauriol J, Alcaide-Loridan C and Middendorp S: Nanog and Oct4 overexpression increases motility and transmigration of melanoma cells. J Cancer Res Clin Oncol 138: 1145-1154, 2012.

11. Tang QL, Zhao ZQ, Li JC, Liang Y, Yin JQ, Zou CY, Xie XB, Zeng YX, Shen JN, Kang T and Wang J: Salinomycin inhibits osteosarcoma by targeting its tumor stem cells. Cancer Lett 311 $113-121,2011$.

12. Martins-Neves SR, Lopes ÁO, do Carmo A, Paiva AA, Simões PC, Abrunhosa AJ and Gomes CM: Therapeutic implications of an enriched cancer stem-like cell population in a human osteosarcoma cell line. BMC Cancer 12: 139, 2012.

13. Huang Y, Dai H and Guo QN: TSSC3 overexpression reduces stemness and induces apoptosis of osteosarcoma tumor-initiating cells. Apoptosis 17: 749-761, 2012.

14. Liu Q, Huang J, Zhou N, Zhang Z, Zhang A, Lu Z, Wu F and Mo YY: LncRNA loc285194 is a p53-regulated tumor suppressor. Nucleic Acids Res 41: 4976-4987, 2013

15. Isin M and Dalay N: LncRNAs and neoplasia. Clin Chim Acta 444: 280-288, 2015

16. Bauderlique-Le Roy H, Vennin C, Brocqueville G, Spruyt N, Adriaenssens E and Bourette RP: Enrichment of human stem-like prostate cells with s-SHIP promoter activity uncovers a role in stemness for the long noncoding RNA H19. Stem Cells Dev 24: 1252-1262, 2015.

17. Shahryari A, Rafiee MR, Fouani Y, Oliae NA, Samaei NM Shafiee M, Semnani S, Vasei M and Mowla SJ: Two novel splice variants of SOX2OT, SOX2OT-S1 and SOX2OT-S2 are coupregulated with SOX2 and Oct4 in esophageal squamous cell carcinoma. Stem Cells 32: 126-134, 2014.

18. Chinese National Institutes of Health: Ethics examination in biomedicine studies involving the human body. Chinese National Institutes of Health, Beijing, China, 2007.

19. Hu J, Fang Y, Cao Y, Qin R and Chen Q: miR-449a regulates proliferation and chemosensitivity to cisplatin by targeting cyclin D1 and BCL2 in SGC7901 cells. Dig Dis Sci 59: 336-345, 2014.

20. Wang L, Wang J, Wang Y, Fu Q, Lei YH, Nie ZY, Qiu J and Bao TY: Protective effect of exogenous matrix metalloproteinase-9 on chronic renal failure. Exp Ther Med 7: 329-334, 2014
21. Livak KJ and Schmittgen TD: Analysis of relative gene expression data using real-time quantitative PCR and the 2(-Delta Delta C(T)) Method. Methods 25: 402-408, 2001.

22. Lee SH, Koo BS, Kim JM, Huang S, Rho YS, Bae WJ, Kang HJ, Kim YS, Moon JH and Lim YC: Wnt/ $\beta$-catenin signalling maintains self-renewal and tumourigenicity of head and neck squamous cell carcinoma stem-like cells by activating October4. J Pathol 234: 99-107, 2014.

23. Xiao H, Jiang N, Zhou B, Liu Q and Du C: TAZ regulates cell proliferation and epithelial-mesenchymal transition of human hepatocellular carcinoma. Cancer Sci 106: 151-159, 2015.

24. Vaiphei K, Sinha SK and Kochhar R: Comparative analysis of Oct4 in different histological subtypes of esophageal squamous cell carcinomas in different clinical conditions. Asian Pac J Cancer Prev 15: 3519-3524, 2014.

25. Li N, Deng W, Ma J, Wei B, Guo K, Shen W, Zhang Y and Luo S: Prognostic evaluation of nanog, Oct4, Sox2, PCNA, Ki67 and E-cadherin expression in gastric cancer. Med Oncol 32: 433, 2015.

26. Chen ZS, Ling DJ, Zhang YD, Feng JX, Zhang XY and Shi TS: Octamer-binding protein 4 affects the cell biology and phenotypic transition of lung cancer cells involving $\beta$-catenin/E-cadherin complex degradation. Mol Med Rep 11: 1851-1858, 2015.

27. Jóźwicki W, Brożyna AA and Siekiera J: Expression of Oct4A: The first step to the next stage of urothelial bladder cancer progression. Int J Mol Sci 15: 16069-16082, 2014.

28. Shen L, Qin K, Wang D, Zhang Y, Bai N, Yang S, Luo Y, Xiang R and Tan X: Overexpression of Oct4 suppresses the metastatic potential of breast cancer cells via Rnd1 downregulation. Biochim Biophys Acta 1842: 2087-2095, 2014.

29. Li JP, Liu LH, Li J, Chen Y, Jiang XW, Ouyang YR, Liu YQ, Zhong $\mathrm{H}, \mathrm{Li} \mathrm{H}$ and Xiao T: Microarray expression profile of long noncoding RNAs in human osteosarcoma. Biochem Biophys Res Commun 433: 200-206, 2013.

30. Dong Y, Liang G, Yuan B, Yang C, Gao R and Zhou X: MALAT1 promotes the proliferation and metastasis of osteosarcoma cells by activating the PI3K/Akt pathway. Tumour Biol 36: 1477-1486, 2015.

31. Zhang Q, Geng PL, Yin P, Wang XL, Jia JP and Yao J: Down-regulation of long non-coding RNA TUG1 inhibits osteosarcoma cell proliferation and promotes apoptosis. Asian Pac J Cancer Prev 14: 2311-2315, 2013.

32. Pasic I, Shlien A, Durbin AD, Stavropoulos DJ, Baskin B, Ray PN, Novokmet A and Malkin D: Recurrent focal copy-number changes and loss of heterozygosity implicate two noncoding RNAs and one tumor suppressor gene at chromosome $3 \mathrm{q} 13.31$ in osteosarcoma. Cancer Res 70: 160-171, 2010.

33. Wang Y, Yao J, Meng H, Yu Z, Wang Z, Yuan X, Chen H and Wang A: A novel long non-coding RNA, hypoxia-inducible factor-2 $\alpha$ promoter upstream transcript, functions as an inhibitor of osteosarcoma stem cells in vitro. Mol Med Rep 11: 2534-2540, 2015. 